\title{
CONTROLE DE PLANTAS DANINHAS NA CULTURA DO MILHO POR MEIO DE HERBICIDAS APLICADOS EM PRÉ-EMERGÊNCIA ${ }^{1}$
}

\author{
Hugo de Almeida Dan², Alberto Leão de Lemos Barroso ${ }^{3}$,
} Lilian Gomes de Moraes Dan², Thiago Rezende Finotti ${ }^{3}$, Cleriston Feldkircher ${ }^{3}$, Vanessa Soares Santos ${ }^{3}$

\begin{abstract}
WEED CONTROL IN MAIZE

BY USING PRE-EMERGENCE HERBICIDES

This study aimed to estimate the effectiveness of weed control in maize (Zea mays L.), under a no-till system, in the Cerrado (Brazilian Savanna) region, by applying pre-emergence herbicides. Two experiments were carried out in Montevidiu, Goiás State, Brazil, during the 2007/2008 crop, in a randomized block design, with four replications. The following treatments were evaluated: atrazine $\left(1,600 \mathrm{~g} \mathrm{ha}^{-1}\right)$, atrazine $+\mathrm{s}$-metolachor $\left(1,665 \mathrm{~g} \mathrm{ha}^{-1}+1,305 \mathrm{~g} \mathrm{ha}^{-1}\right)$, s-metolachor $\left(1,680 \mathrm{~g} \mathrm{ha}^{-1}\right)$, atrazine + simazine $\left(250 \mathrm{~g} \mathrm{ha}^{-1}+250 \mathrm{~g} \mathrm{ha}^{-1}\right)$, and control with and without weeds. It was found out that, 28 days after the treatments application, the herbicides atrazine and s-metolachor were not effective in controlling Cenchrus echinatus and Alternanthera tenella, respectively. The associations between atrazine + s-metolachor and atrazine + simazine have provided significant increases in the Euphorbia heterophilla and Alternanthera tenella control. The presence of weeds had a negative effect on crop yield.
\end{abstract}

KEY-WORDS: Zea mays; atrazine; s-metolachor; simazine; no-tillage.

\section{INTRODUÇÃO}

O milho destaca-se como uma das principais culturas na região dos Cerrados, cultivado em pequenas, médias e grandes propriedades (Oliveira et al. 2009). Dentre os fatores que influenciam na produtividade da cultura, destaca-se a interferência de plantas daninhas, durante o período de maior vulnerabilidade da cultura (Vidal et al. 2005, Silva et al. 2007).

O controle químico de plantas daninhas, na cultura do milho, tem se destacado pela eficácia, rendimento operacional e melhor relação custo/bene-

\section{RESUMO}

Este trabalho teve por objetivo estimar a eficácia do controle de plantas daninhas na cultura do milho (Zea mays L.), cultivado em sistema plantio direto, em região de Cerrado, por meio da aplicação, em pré-emergência, de herbicidas. Foram realizados dois ensaios, no município de Montevidiu (GO), durante a safra 2007/2008, dispostos em delineamento de blocos ao acaso, com quatro repetições. Foram avaliados os seguintes tratamentos: atrazine $\left(1.600 \mathrm{~g} \mathrm{ha}^{-1}\right)$, atrazine $+\mathrm{s}$-metolachor (1.665 $\left.\mathrm{g} \mathrm{ha}^{-1}+1.305 \mathrm{~g} \mathrm{ha}^{-1}\right)$, s-metolachor (1.680 $\left.\mathrm{g} \mathrm{ha}^{-1}\right)$, atrazine + simazine $\left(250 \mathrm{~g} \mathrm{ha}^{-1}+250 \mathrm{~g} \mathrm{ha}^{-1}\right)$ e testemunha com e sem a presença de plantas daninhas. Aos 28 dias após a aplicação dos tratamentos, constatou-se que os herbicidas atrazine e s-metolachor não foram eficientes no controle de Cenchrus echinatus e Alternanthera tenella, respectivamente. As associações entre os herbicidas atrazina + s-metolachor e atrazine + simazine proporcionaram incrementos significativos no controle de Euphorbia heterophilla e Alternanthera tenella. A presença de plantas daninhas influenciou negativamente na produtividade da cultura.

PALAVRAS-CHAVE: Zea mays; atrazine; s-metolachor; simazine; plantio direto.

fício. A eficácia de controle é variável e dependente das características físico-químicas do produto, condições edafoclimáticas, época de aplicação e espécies de plantas daninhas a serem controladas (Merotto Jr. et al. 1997).

A utilização de herbicidas pré-emergentes, com efeito residual prolongado, tem sido um dos fatores associados à elevada eficácia no controle de plantas daninhas, durante o período crítico de competição (Monquero et al. 2008). Para estes herbicidas, ressalta-se a importância do tipo, textura e umidade do solo e a presença de restos vegetais, já que estes possuem influência marcante na dinâmica

1. Trabalho recebido em abr./2009 e aceito para publicação em out./2010 (nº registro: PAT 6057/ DOI: 10.5216/pat.v40i4.6057).

2. Universidade Estadual de Maringá, Núcleo de Estudos Avançados em Ciência das Plantas Daninhas, Maringá, PR, Brasil.

E-mails: halmeidadan@gmail.com, lilian-agronoma@hotmail.com.

3. Universidade de Rio Verde, Faculdade de Agronomia, Departamento de Fitotecnia, Rio Verde, GO, Brasil.

E-mails: all_barroso@hotmail.com,tfinotti@yahoo.com,cleriston_agro@yahoo.com.br, vanessaagronoma@gmail.com. 
de herbicidas no ambiente. Abdelhafid et al. (2000) citam que solos de textura argilosa e elevados teores de matéria orgânica apresentam maior capacidade de retenção de herbicidas, necessitando de maiores ajustes, principalmente quando o interesse está na atividade residual do herbicida. Fontes et al. (2010) relatam a importância do estudo da dose do herbicida, em relação à textura do solo, para o controle de plantas daninhas, em diferentes sistemas de cultivo.

Com relação ao posicionamento de doses, em diferentes sistemas de cultivo, Sharma et al. (2000) e Scivittaro et al. (2005) observaram que a utilização de $2,5 \mathrm{~kg} \mathrm{ha}^{-1}$ de atrazine proporcionou excelentes níveis de controle de Echinochloa colonum, quando aplicado em pré-emergência, em solo de textura média, manejado convencionalmente. A associação de atrazine e metolachlor com o herbicida RPA 201772, aplicados em pré-emergência do milho, trouxe incrementos positivos no controle de Setaria faberi, Amaranthus hybridus e Chenopodium album, quando estes herbicidas foram utilizados em cultivo convencional (Young et al. 1999). Theisen et al. (2000), visando ao controle de papua (Brachiaria plantaginea), notaram que a dose de $3,0 \mathrm{~kg} \mathrm{ha}^{-1}$ de atrazine apresentou $82 \%$ de eficácia de controle desta espécie, quando aplicado logo após a semeadura. Para Brighenti et al. (1998), o atrazine foi eficiente no controle de Sida rhombifolia, entretanto, não controlou, eficientemente, a Brachiaria plantaginea. Já o herbicida metolachlor controlou, satisfatoriamente, além da B. plantaginea, as espécies Cenchrus echinatus, Digitaria horizontalis e Commelina benghalensis. Ao associar os herbicidas atrazine e metolachlor, Brighenti et al. (1998) e Barros et al. (2000) obtiveram maior espectro de controle das plantas daninhas.

Com o advento do plantio direto e do cultivo mínimo, a utilização de herbicidas pré-emergentes tem sido relegada, na região dos Cerrados, principalmente devido a questões operacionais e ao reduzido número de informações referentes ao comportamento dos herbicidas sobre o controle das principais plantas daninhas, em sistemas de manejos utilizados na região do Cerrado.

Este trabalho teve por objetivo avaliar a eficácia de herbicidas aplicados em pré-emergência da cultura do milho (Zea mays L.), cultivado em sistema plantio direto, na região do Cerrado.

\section{MATERIAL E MÉTODOS}

Dois experimentos foram realizados no município de Montevidiu $\left(17^{\circ} 26^{\prime} 35,5^{\prime}\right.$ 'S, $51^{\circ} 15^{\prime} 15,5^{\prime \prime} \mathrm{W}$ e $810 \mathrm{~m}$, para o ensaio 1 , e $17^{\circ} 25^{\prime} 39,1^{\prime \prime} \mathrm{S}, 51^{\circ} 14^{\prime} 12,1$ ' 'W e $790 \mathrm{~m}$, para o ensaio 2), Estado de Goiás, durante a safra 2007/2008.

A semeadura do híbrido Pioneer 30F35 foi realizada sobre um Latossolo Vermelho-Amarelo distroférrico típico, com $320 \mathrm{~g} \mathrm{~kg}^{-1}$ e $360 \mathrm{~g} \mathrm{~kg}^{-1}$ de argila e $2,1 \%$ e $2,4 \%$ de matéria orgânica, para os ensaios 1 e 2 , respectivamente. A adubação de plantio, de ambos os experimentos, foi de $400 \mathrm{~kg} \mathrm{ha}^{-1} \mathrm{da}$ fórmula 8-20-10+2\% de zinco, mais $200 \mathrm{~kg} \mathrm{ha}^{-1} \mathrm{de}$ ureia e $75 \mathrm{~kg} \mathrm{ha}^{-1}$ de cloreto de potássio em cobertura, aos 20 e 35 dias após a emergência. Ambos os solos apresentaram teores médios de $\mathrm{Zn}$, de acordo com Sousa \& Lobato (2002).

As sementes de milho foram previamente tratadas com os produtos Cropstar ${ }^{\circledR}(300 \mathrm{~mL} / 100 \mathrm{~kg}$ de sementes $)$ e Derosal Plus ${ }^{\circledR}(200 \mathrm{~mL} / 100 \mathrm{~kg}$ de sementes). A semeadura foi realizada em área de sistema de plantio direto, formada pela dessecação da vegetação daninha que cobria o solo. O manejo desta área foi realizado com $1.680 \mathrm{~g} \mathrm{ha}^{-1}$ do herbicida glyphosate, sete dias antes da semeadura do milho.

$\mathrm{O}$ delineamento experimental utilizado foi o de blocos ao acaso, composto por seis tratamentos (Tabela 1), com quatro repetições. As dimensões da parcela experimental foram $6,0 \mathrm{~m}$ de comprimento por 4,0 $\mathrm{m}$ de largura (8 linhas de milho, de $0,5 \mathrm{~m}$ ), totalizando $24,0 \mathrm{~m}^{2}$. Obteve-se uma população final de 55 mil plantas de milho por hectare.

A aplicação dos herbicidas foi realizada 24 horas após a semeadura da cultura, por meio de pulverizador costal com pressurização por $\mathrm{CO}_{2}$, munido de pontas de pulverização do tipo AI 110-02, aplicando-se volume equivalente a $150 \mathrm{~L} \mathrm{ha}^{-1}$ de calda. As condições ambientais, no momento da aplicação, respeitaram as condições exigidas. $\mathrm{Na}$ ocasião da

Tabela 1. Tratamentos avaliados para o controle de plantas daninhas na cultura do milho (Montevidiu, GO, safra 2007/2008).

\begin{tabular}{lccc}
\hline \multicolumn{1}{c}{ Nome comum* } & Nome comercial** & $\begin{array}{c}\text { Dose } \\
\text { g i.a. ha }\end{array}$ & $\begin{array}{c}\text { Dose } \\
\text { g ou L p.c. }{ }^{-1} \text { ha }^{-1}\end{array}$ \\
\hline atrazine & Gesaprim 500 & 1.600 & 3,2 \\
atrazine + s-metolachor & Primestra Gold & $1.665+1.305$ & 4,5 \\
s-metolachor & Dual Gold & 1.680 & 1,75 \\
[atrazine + simazine] & Primatop SC & $250+250$ & $7+7$ \\
testemunha capinada & - & - & - \\
testemunha infestada & - & - & - \\
\hline * Ingrediente ativo; ** Produto comercial. & &
\end{tabular}


aplicação, o solo da área experimental se encontrava com $70 \%$ da capacidade de campo. No momento da aplicação, grande parte dos restos vegetais (proporcionada pelas plantas daninhas) já estava em pleno processo de decomposição e, aos 15 dias após a aplicação dos tratamentos, o solo encontrava-se completamente descoberto (baixa presença de resíduos vegetais). As condições pluviométricas, durante o período de condução do experimento, foram normais, com regime de chuvas bem distribuído.

As avaliações de controle das plantas daninhas foram realizadas de forma visual, utilizando-se escala percentual de $0 \%$ a $100 \%$, onde $0 \%$ representa ausência de sintomas e $100 \%$ morte de todas as plantas, aos 7, 21 e 28 dias após a emergência (DAE) (Alam 1974). Aos 28 DAE, realizou-se levantamento fitossociológico, por meio de um quadrado de $0,5 \mathrm{~m}^{2}$, quantificando-se as espécies daninhas presentes nas testemunhas.

As avaliações de controle foram realizadas aos 28 DAE. Ao final do ciclo da cultura, avaliou-se o peso de 1.000 grãos e a produtividade, estimados por meio da colheita de $4,5 \mathrm{~m}^{2}$ de área útil (2 linhas centrais de cada parcela de milho). O peso das amostras foi corrigido para $13 \%$.

Após a coleta e tabulação dos dados, procedeu-se à análise de variância, sendo as médias das variáveis significativas comparadas pelo teste de Scott Knott, a 5\% de significância.

\section{RESULTADOS E DISCUSSÃO}

Por meio do levantamento fitossociológico, realizado aos 28 dias após emergência da cultura, observaram-se as seguintes plantas daninhas: ensaio 1: 2 plantas $\mathrm{m}^{2}$ de corda-de-viola (Ipomoea grandifolia), 4,2 plantas $\mathrm{m}^{2}$ de trapoeraba (Commelina benghalensis), 92 plantas $\mathrm{m}^{2}$ de timbete (Cenchrus echinatus) e 3,5 plantas $\mathrm{m}^{2}$ de Leiteiro (Euphorbia heterophilla; ensaio 2: 20,3 plantas $\mathrm{m}^{2}$ de apaga fogo (Alternanthera tenella), 4,5 plantas $\mathrm{m}^{2}$ de capim amargoso (Digitaria insularis), 2,8 plantas $\mathrm{m}^{2}$ de picão preto (Bidens pilosa) e 12,7 plantas $\mathrm{m}^{2}$ de erva de Santa Luzia (Chamaesyce hirta).

Durante a condução do ensaio, não foram observados sintomas de fitointoxicação dos herbicidas sobre a cultura do milho. Segundo Viger et al. (1991), existe correlação direta entre o nível de injúria e o aumento na dose de herbicidas como o metolachlor, porém a toxicidade é maior nas plantas crescidas, em condições de elevada umidade do solo e submetidas a baixa temperatura, condições que não foram observadas no presente ensaio.

Com relação à eficácia dos herbicidas sobre as plantas daninhas, inicialmente, aos 7 dias após a aplicação (DAA), observou-se elevado controle das espécies daninhas presentes nas unidades experimentais (dados não apresentados). Este resultado é de suma importância, pois evita a competição inicial. Os níveis de controle foram reduzidos gradativamente, conforme o aumento do intervalo de tempo entre a aplicação e as avaliações. Aos 28 dias após a aplicação (DAA), ou seja, 24 dias após a emergência da cultura (Tabela 2), os níveis de controle de Ipomoea grandifolia foram semelhantes para os tratamentos atrazine + s-metolachor, atrazine e s-metolachor, que apresentaram $78 \%, 73 \%$ e $73 \%$ de controle, respectivamente. A associação de atrazine + simazine proporcionou controle de $87 \%$ de I. grandifolia, destacando-se perante os demais tratamentos, evi-

Tabela 2. Níveis de controle das plantas daninhas na cultura do milho, aos 28 DAE, no ensaio 1 (Montevidiu, GO, safra 2007/2008).

\begin{tabular}{|c|c|c|c|c|c|}
\hline \multirow[t]{2}{*}{ Tratamentos } & \multirow{2}{*}{$\begin{array}{c}\text { Dose } \\
\text { g i.a. } \text { ha }^{-1}\end{array}$} & \multicolumn{4}{|c|}{ Controle $(\%)$} \\
\hline & & IAOGR $^{2}$ & $\mathrm{COMBE}^{3}$ & $\mathrm{CECHC}^{4}$ & $\mathrm{EPHHL}^{5}$ \\
\hline atrazine & 1.600 & $73,7 b^{1}$ & $82,5 \mathrm{~b}$ & $57,5 \mathrm{~b}$ & $68,7 \mathrm{~b}$ \\
\hline atrazine + s-metolachor & $1.665+1.305$ & $78,7 \mathrm{~b}$ & $76,2 \mathrm{~b}$ & $70,0 \mathrm{c}$ & $82,5 \mathrm{c}$ \\
\hline s-metolachor & 1.680 & $73,7 \mathrm{~b}$ & $83,7 \mathrm{c}$ & $72,5 \mathrm{c}$ & $60 \mathrm{~b}$ \\
\hline [atrazine + simazine $]$ & $250+250$ & $87,5 \mathrm{c}$ & $88,7 \mathrm{c}$ & $68,7 \mathrm{c}$ & $87,5 \mathrm{c}$ \\
\hline testemunha infestada & - & 0,0 a & 0,0 a & 0,0 a & 0,0 a \\
\hline testemunha capinada & & $100 \mathrm{~d}$ & $100 \mathrm{~d}$ & $100 \mathrm{~d}$ & $100 \mathrm{~d}$ \\
\hline CV\% & & 7,0 & 7,85 & 10,09 & 7,99 \\
\hline
\end{tabular}

${ }^{1}$ Médias de tratamentos seguidas de mesma letra não diferem entre si, pelo teste de Scott Knott, a 5\% de Probabilidade; ${ }^{2}$ IAOGR: Corda-de-viola (Ipomoea grandifolia);

${ }^{3}$ COMBE: Trapoeraba (Commelina benghalensis); ${ }^{4}$ CECHC: Timbete (Cenchrus echinatus); ${ }^{5}$ EPHHL: Leiteiro (Euphorbia heterophilla). 
denciando que a associação entre os dois herbicidas inibidores do fotossistema II foi positiva no controle desta espécie.

Commelina benghalensis apresentou maior sensibilidade aos herbicidas atrazine + simazine e s-metolachlor, que obtiveram controle superior a $83 \%$. Dourado Neto et al. (2003) observaram que a associação entre os herbicidas atrazine + alachlor não controlou, de maneira eficiente, esta espécie. Utilizando 2,4 $\mathrm{kg} \mathrm{ha}^{-1}$ de metolachlor, Brighenti et al. (1998) obtiveram satisfatórios níveis de controle de C. benghalensis, corroborando os resultados do presente ensaio. Segundo Rodrigues \& Almeida (2005), o metolachlor é efetivo no controle de algumas monocotiledôneas, como gramíneas, e C. benghalensis, espécies frequentemente observadas em cultivos no Cerrado.

Os herbicidas não apresentaram controle satisfatório ( $<80 \%$ ) de Cenchrus echinatus, aos 28 DAA. Durante este período, observou-se, por meio de contagem, população de 98 plantas $\mathrm{m}^{2}$ desta espécie. Apesar de o s-metolachor suprimir cerca de 72,5\% das plantas presentes nas unidades experimentais, este não diferiu das associações entre atrazina + s-metolachlor e atrazine + simazine, com $70 \%$ e $68 \%$ de controle, respectivamente. Estes resultados se devem às elevadas densidades populacionais desta planta daninha (92 plantas $\mathrm{m}^{2}$ ). Brighenti et al. (1998) obtiveram sucesso no controle em elevadas pressões populacionais, quando utilizaram $3,5 \mathrm{~kg} \mathrm{ha}^{-1}$ de metolachlor, o que corresponte a mais do que o dobro da dose utilizada no presente estudo. Dourado Neto et al. (2003) constataram que o metolachlor e suas associações foram eficientes no controle desta espécie. Ao avaliar o herbicida acetochlor sobre o C. echinatus,
Martini et al. (2005) observaram que este herbicida apresentou excelente efeito residual, ao ser utilizado em pós-emergência precoce, na cultura do milho. A dose utilizada do herbicida atrazine não proporcionou controle satisfatório de C. echinatus, corroborando os resultados apresentados por Brighenti et al. (1998). Fato semelhante pôde ser observado em plantas de E. heterophilla, que obtiveram apenas $68 \%$ de controle, durante a última avaliação.

As associações entre os herbicidas atrazine + simazine e atrazina $+\mathrm{s}$-metolachor proporcionaram incrementos significativos no controle de $E$. heterophilla (Tabela 2). Já s-metholaclor não apresentou controle satisfatório desta espécie, não diferindo de atrazine, quando utilizado de forma isolada.

Outra espécie avaliada foi o Apaga fogo (Alternanthera tenella), planta daninha muito frequente nas áreas cultivadas do Cerrado brasileiro. Considerando-se os herbicidas pré-emergentes avaliados no ensaio 2 (Tabela 3), as associações entre atrazina + s-metolachor e atrazine + simazine promoveram controle de $93 \%$ e $92 \%$, respectivamente, 28 dias após a emergência da cultura. Estes tratamentos proporcionaram incrementos significativos, em relação aos herbicidas utilizados de forma isolada, diferindo dos resultados encontrados por Dourado Neto et al. (2003), ao observar que a associação atrazina + s-metolachor controlou apenas $67 \%$ desta espécie.

O capim Amargoso (Digitaria insularis) apresentou maior sensibilidade aos tratamentos s-metolachor e atrazine + s-metolachor (Tabela 3). Estes apresentaram níveis de controle de $93 \%$ e $90 \%$ e foram significativamente superiores aos demais tratamentos, não diferindo, apenas, da testemunha capinada. Já o atrazine, de forma isolada, não foi

Tabela 3. Níveis de controle das plantas daninhas na cultura do milho, aos 28 DAE, no ensaio 2 (Montevidiu, GO, safra 2007/2008).

\begin{tabular}{|c|c|c|c|c|c|}
\hline \multirow[t]{2}{*}{ Tratamentos } & \multirow{2}{*}{$\begin{array}{c}\text { Dose } \\
\text { g i.a. ha-1 }\end{array}$} & \multicolumn{4}{|c|}{ Controle $(\%)$} \\
\hline & & ALRT $^{2}$ & DIGIN $^{3}$ & $\mathrm{BDPI}^{4}$ & $\mathrm{EPHHI}^{5}$ \\
\hline atrazine & 1.600 & $82,7 \mathrm{c}^{1}$ & $71,2 \quad b$ & $94,7 \mathrm{~d}$ & $98,2 \mathrm{c}$ \\
\hline atrazine + s-metolachor & $1.665+1.305$ & $93,5 \mathrm{~d}$ & $93,2 \mathrm{~d}$ & $90,7 \mathrm{c}$ & $97,5 \mathrm{c}$ \\
\hline s-metolachor & 1.680 & $75,5 \mathrm{~b}$ & $90,7 \mathrm{~d}$ & $71,5 \mathrm{~b}$ & $58,7 \mathrm{~b}$ \\
\hline [atrazine + simazine] & $250+250$ & $92,5 \mathrm{~d}$ & $82,0 \quad \mathrm{c}$ & $96,0 \mathrm{~d}$ & $94,0 \mathrm{c}$ \\
\hline testemunha infestada & - & 0,0 a & 0,0 a & 0,0 a & 0,0 a \\
\hline testemunha capinada & & $100 \mathrm{e}$ & $100 \mathrm{~d}$ & $100 \mathrm{~d}$ & $100 \mathrm{c}$ \\
\hline $\mathrm{CV} \%$ & & 4,64 & 4,76 & 6,03 & 3,66 \\
\hline
\end{tabular}

${ }^{1}$ Médias de tratamentos seguidas de mesma letra não diferem entre si, pelo teste de Scott Knott, a 5\% de probabilidade; ${ }^{2}$ ALRT: Apaga fogo (Alternanthera tenella); ${ }^{3}$ DIGIN: Amargoso (Digitaria insularis); ${ }^{4}$ BDPI: Picão preto (Bidens pilosa); ${ }^{5}$ EPHHI: Erva Santa Luzia (Chamaesyce hirta). 
Tabela 4. Produtividade de grãos de milho, após a aplicação de diversos tratamentos, nos ensaios 1 e 2 (Montevidiu, GO, safra 2007/2008).

\begin{tabular}{|c|c|c|c|c|c|c|}
\hline \multirow[t]{2}{*}{ Tratamentos } & \multirow[t]{2}{*}{$\begin{array}{c}\text { Dose } \\
\text { g i.a. ha }{ }^{-1}\end{array}$} & \multicolumn{2}{|c|}{ Peso de 1.000 grãos (g) } & \multicolumn{3}{|c|}{ Produtividade de grãos $\left(\mathrm{kg} \mathrm{ha}^{-1}\right)$} \\
\hline & & 1 & 2 & 1 & 2 & \\
\hline atrazine & 1.600 & $273 \mathrm{a}^{*}$ & $272 \mathrm{a}$ & $6320 \mathrm{a}$ & 8006 & $\mathrm{a}$ \\
\hline atrazine + s-metolachor & $1.665+1.305$ & 276 a & 276 a & $6678 \mathrm{a}$ & 9244 & $\mathrm{a}$ \\
\hline s-metolachor & 1.680 & 276 a & 274 a & 6719 a & 7685 & $\mathrm{a}$ \\
\hline [atrazine + simazine] & $250+250$ & $277 \mathrm{a}$ & 269 a & $6819 \mathrm{a}$ & 7931 & $\mathrm{a}$ \\
\hline testemunha infestada & - & $264 \mathrm{a}$ & 264 a & $5536 \mathrm{~b}$ & 6580 & $\mathrm{~b}$ \\
\hline testemunha capinada & - & 276 a & 279 a & $7164 \mathrm{a}$ & 8635 & $\mathrm{a}$ \\
\hline $\mathrm{CV} \%$ & - & 3,60 & 8,43 & 14,27 & 9,75 & \\
\hline
\end{tabular}

* Médias de tratamentos seguidas de mesma letra não diferem entre si, pelo teste de Scott Knott, a 5\% de probabilidade.

eficiente no controle desta espécie. Brighenti et al. (1998) obtiveram controle satisfatório de Digitaria horizontalis, com doses de atrazine superiores a $3,0 \mathrm{~kg} \mathrm{ha}^{-1}$.

Constatou-se que atrazine + simazine e atrazine apresentaram 96\% e 94\% de controle do Picão preto (Bidens pilosa), superando os demais herbicidas. Quanto ao s-metolachlor, observou-se controle duvidoso (71\%), corroborando os resultados encontrados por Laca-buendía (2005).

No caso da Erva Santa Luzia (Chamaesyce hirta), somente os tratamentos à base de s-metolachor e atrazine (isolados) proporcionaram controle insuficiente desta espécie. Os demais herbicidas pré-emergentes foram eficientes, mantendo a cultura com baixos níveis de presença da espécie, aos 28 dias após a aplicação.

A presença de plantas daninhas proporcionou redução de $22 \%$ e $23 \%$ na produtividade da cultura, quando a comparação é efetuada entre as testemunhas (Tabela 4). Estes valores representam o primeiro e segundo ensaios, respectivamente. No entanto, esta diferença foi reduzida entre os tratamentos herbicidas.

Apesar dos diferentes níveis de controle apresentados pelos herbicidas, não foram observadas diferenças significativas entre estes tratamentos, com relação à produtividade da cultura. Todavia, todos os tratamentos diferiram da testemunha infestada. Resultados semelhantes foram encontrados por Ford \& Pleasant (1994) e Silva et al. (2004), ao evidenciarem que, além da produtividade, a interferência pode influenciar, também, os componentes do rendimento da cultura, como o peso de 1.000 grãos, fato não observado no presente ensaio. É importante salien- tar que o controle inadequado das plantas daninhas pode ocasionar a reinfestação da área, aumentando, consequentemente, o estoque de sementes no solo, causando danos indiretos à colheita, justificando a utilização do período total de prevenção da interferência.

\section{CONCLUSÕES}

1. Os herbicidas atrazine e s-metolachor não foram eficientes no controle de Cenchrus echinatus e Alternanthera tenella. Entretanto, a associação atrazine + simazine apresentou eficiente controle para I. grandifolia, C. benghalensis, E. heterophilla e C. echinatus.

2. As associações entre os herbicidas atrazina + s-metolachor e atrazine + simazine proporcionaram incrementos no controle de Euphorbia heterophilla e Alternanthera tenella.

3. A interferência causada pelas plantas daninhas influenciou negativamente na produtividade da cultura. Contudo, apesar dos diferentes níveis de controle, não foram observadas diferenças significativas entre os tratamentos e a testemunha capinada.

\section{REFERÊNCIAS}

ABDELHAFID, R.; HOUOT, S.; BARRIUSO, E. Dependence of atrazine degradation on $\mathrm{C}$ and $\mathrm{N}$ availability in adapted and non-adapted soils. Soil Biology and Biochemistry, New York, v. 32, n. 3, p. 389-401, 2000. 
ASOCIACIÓN LATINOAMERICANA DE MALEZAS (Alam). Recomendaciones sobre unificación de los sistemas de evaluación en ensayos de control de malezas. Alam, Bogotá, v. 1, n. 1, p. 35-38, 1974.

BARROS, A. C.; UEDA, A.; SCHUMM, K. C. Efeito de herbicidas de pós-emergência, aplicados em várias épocas, comparados com atrazine + metolachlor, em préemergência, na cultura do milho. Revista Brasileira de Herbicidas, Passo fundo, v. 1, n. 3, p. 207-212, 2000.

BRIGHENTI, A. M. et al. Controle químico de plantas daninhas em cultivos sucessivos de milho e feijão. Planta Daninha, Botucatu, v. 16, n. 2, p. 109-116, 1998.

DOURADO NETO, D. et al. Morfologia e produtividade em milho, afetado por herbicidas em duas épocas de aplicação. Revista da FZVA, Uruguaiana, v. 10, n. 1, p. 82-94, 2003.

FONTES, J. R. A.; GONÇALVES, J. R. P.; MORAIS, R. R. Tolerância do feijão-caupi ao herbicida oxadiazon. Pesquisa Agropecuária Tropical, Goiânia, v. 40, n. 1, p. 122-129, 2010.

FORD, G. T.; PLEASANT, J. Competitive abilities of six corn (Zea mays L.) hybrids with four weed control practices. Weed Technology, Champaign, v. 8, n. 1, p. 124128, 1994.

LACA-BUENDÍA, J. P. Seletividade e eficácia das formulações com os herbicidas atrazine/dimethenamid-p e atrazine/dimethenamid, no controle de plantas daninhas na cultura do milho. Revista Brasileira de Herbicidas, Passo Fundo, v. 4, n. 1, p. 9-17, 2005.

MARTINI, G.; PEDRINHO JÚNIOR, A. F. F.; DURIGAN, J. Z. Influência da época de controle das plantas daninhas, sobre a eficácia do herbicida acetochlor em milho. Revista Brasileira de Herbicidas, Passo Fundo, v. 2, n. 1, p. $315-$ 321, 2005.

MEROTTO JÚNIOR, A. et al. Aumento da população de plantas e uso de herbicidas no controle de plantas daninhas em milho. Planta Daninha, Viçosa, v. 15, n. 2, p. 141-151, 1997.

MONQUERO, P. A. et al. Eficiência de herbicidas préemergentes após períodos de seca. Planta daninha, Viçosa, v. 26, n. 1, p. 185-193, 2008.

OLIVEIRA, M. N. et al. Efeitos da introdução do sistema de plantio direto de milho por agricultores familiares do município de Unaí, MG (Cerrado brasileiro). Pesquisa Agropecuária Tropical, Goiânia, v. 39, n. 1, p. 51-60, 2009.
RODRIGUES, B. N.; ALMEIDA, F. S. Guia de herbicidas. 5. ed. Londrina: Iapar, 2005.

SHARMA, R. P.; DADHEECH, R. C.; JAT, L. N. Effect of atrazine and nitrogen on weed growth and yield of sorghum [Sorghum bicolor (L.) Moench]. Indian Journal of Weed Science, Hisar, v. 32, n. 3, p. 96-97, 2000.

SCIVITTARO, W. B. et al. Doses de nitrogênio e de atrazine em cultivo de sorgo em terras baixas. Revista Brasileira Agrociência, Pelotas, v. 11, n. 3, p. 315-321, 2005.

SILVA, P. S. L. et al. Number and time of weeding effects on maize grain yield. Revista Brasileira de Milho e Sorgo, Sete Lagoas, v. 3, n. 2, p. 204-213, 2004.

SILVA, A. A. et al. Controle de plantas daninhas. Brasília, DF: Associação Brasileira de Educação Agrícola Superior, 2007.

SOUSA, D. M. G. de; LOBATO, E. Calagem e adubação para culturas anuais e semiperenes. In: SOUSA, D. M. G. de; LOBATO, E. (Eds.). Cerrado: correção do solo e adubação. Planaltina: Embrapa Cerrados, 2002. p. 283316.

THEISEN, G. et al. Controle de papuã (Brachiaria plantaginea) com atrazine na cultura do clima temperado. Pelotas: Fufepel, 2000. (Documentos, 70).

VIDAL, R. A.; FLECK, N. G.; MEROTTO JR., A. Período anterior ao dano no rendimento econômico (PADRE): nova abordagem sobre os períodos de interferência entre plantas daninhas e cultivadas. Planta Daninha, Viçosa, v. 23, n. 3, p. 387-396, 2005.

VIGER, P. R.; EBERLEIN, C. V.; FUERST, E. P. Influence of available soil water content, temperature, and CGA154281 on metolachlor injury to corn. Weed Science, Lawrence, v. 39, n. 2, p. 227-231, 1991.

YOUNG, B. G.; HART, S. E.; SIMMONS, F. W. Preemergence weed control in conventional-till corn (Zea mays) with RPA 201772. Weed technology, Champaign, v. 13, n. 3, p. 606-664, 1999. 